\title{
Hybrid optical CDMA and DWDM system implemented under the influence of non-linear effects
}

\author{
Naif Alsowaidi ${ }^{1}$, Tawfig Eltaif ${ }^{2}$, Mohd Ridzuan Mokhtar ${ }^{3}$ \\ ${ }^{1,2}$ Faculty of Engineering \& Technology, Multimedia University, Malaysia \\ ${ }^{3}$ Faculty of Engineering, Multimedia University, Malaysia
}

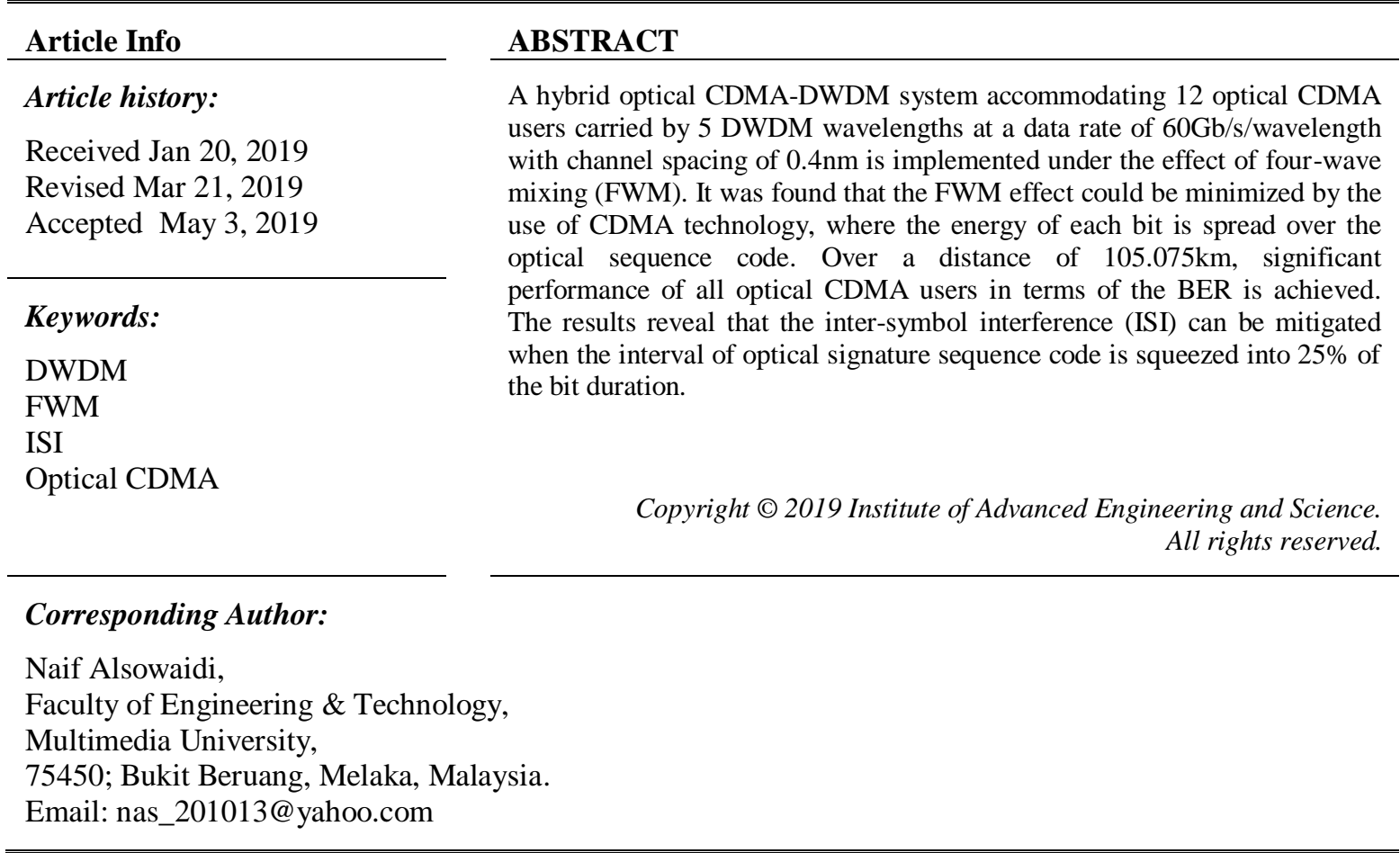

\section{INTRODUCTION}

As data transmission get more and heavier, the need of high rate transmission technology is increasing, the target is a high speed capacity transmission technology. Utilizing the bandwidth of the optical fiber to meet the users demand for high-speed communications such as high speed internet, data exchange like audio and video, images, High Definition Television (HDTV) through network, is one of the main challenges. WDM technology enables efficient usage of fiber capacity by dividing the bandwidth into different wavelength sets, where each wavelength supports communication between a pair of end nodes. Different wavelengths are multiplexed at the transmitter side and carried simultaneously over the same physical fiber. At the receiving end, they are de-multiplexed and each wavelength is directed to the designated receiver. Due to the unique properties of Optical Code Division Multiplexing Access (Optical CDMA) technology such as fully asynchronous access capability, high security and soft capacity, it has been widely researched.

To make full use of the huge bandwidth offered by optical fiber and soft capacity properties of optical CDMA, the convergence of optical CDMA and DWDM can be regarded as a new competitive option for the future optical access network. However, it's well known that in WDM, the maximum allowable transmitted power channel is limited due to nonlinear phenomenon known as four-wave mixing (FWM) effect. The physical origin of FWM is from the third order nonlinear susceptibility of optical fiber, where there are three wavelengths propagate, hence, fourth intermodulation product is produced. On the other hand, multiple access interference (MAI) is the big problem facing the researchers in optical CDMA area, which is caused by the overlapping signaling between the users. Few researches of hybrid systems have been 
conducted in the literature [1-8]. However the effect of FWM has not been addressed yet. In fact FWM effect has been widely investigated in purely WDM systems [9-21].

In this paper, an optical CDMA-DWDM hybrid system with spacing $0.4 \mathrm{~nm}$ is proposed to increase the capacity of the network and reduce the FWM effects. Zero cross correlation (ZCC) code is used as a signature sequence code, which has good properties in terms of auto and cross correlation [22]; hence the MAI can be reduced. On the other hand, the nonlinear effect of FWM, which degrades the signals in DWDM system, can be reduced by CDMA technology. To obtain the useful information for practical system design, the hybrid system has been tested with different values of transmitted power while dispersion compensating fiber (DCF) is used in the transmission line to compensate the dispersion. The paper is organized as follows; section 2 provides a description of the proposed hybrid system, while section 3 presents the analysis of FWM in the hybrid system, where section 4 is devoted for the results and discussions and the conclusion is drawn in section 5 .

\section{SYSTEM DESCRIPTION}

The operational block diagram of the proposed hybrid system is shown in Figure 1, where there are $M$ continuous wave distributed feedback lasers (DFB) to generate $M$ wavelengths with wavelength spacing of $0.4 \mathrm{~nm}$, each followed by an external modulator drives by sinusoidal signal, which used to create a periodic train of return to zero (RZ) pulses, then each wavelength split into $N$ outputs ports, each represents one user's data, where an external modulator is driven by an electrical NRZ data source used to impose the modulation by blocking the zero bits. The NRZ data is the pseudo-random binary sequence (PRBS) with a length of $\left(2^{9}-1\right)$ at the bit rate of 5 Gbps. The modulated data of each user is then encoded by a unique code of Zero Cross-correlation (ZCC). The $N$ encoded users' data are combined together by an optical combiner, where the same optical sequence code will be used for all the other wavelengths. Finally, all the $M$ wavelengths multiplexed by WDM multiplexer and sent over single mode fiber. In addition, dispersion compensating fiber (DCF) is used for dispersion compensation, and preamplifier used to composite the loss. Each wavelength/channel can accommodate $N$ optical CDMA users; where each user is encoded by a unique optical code and the same code can be reused for other wavelengths. So, the proposed hybrid system is capable of accommodating $M \times N$ users. ZCC sequences used as signature code for the optical CDMA users, where there are $N$ zero cross-correlation sequences that can be generated; each sequence code has a weight $w$ and a length $F_{m}$, where the length of the ZCC sequences is $F_{m}=2^{m}\left(w^{2}+w\right), m$ is the mapping process [22]. As mentioned that a splitter is used to split one wavelength into $N$ outputs and each $n^{\text {th }}$ output is modulated by $5 \mathrm{Gbps}$ then encoded by time delays to correlate the desired code. In order to mitigate the ISI, the sequence code interval must be squeezed into less than bit duration.

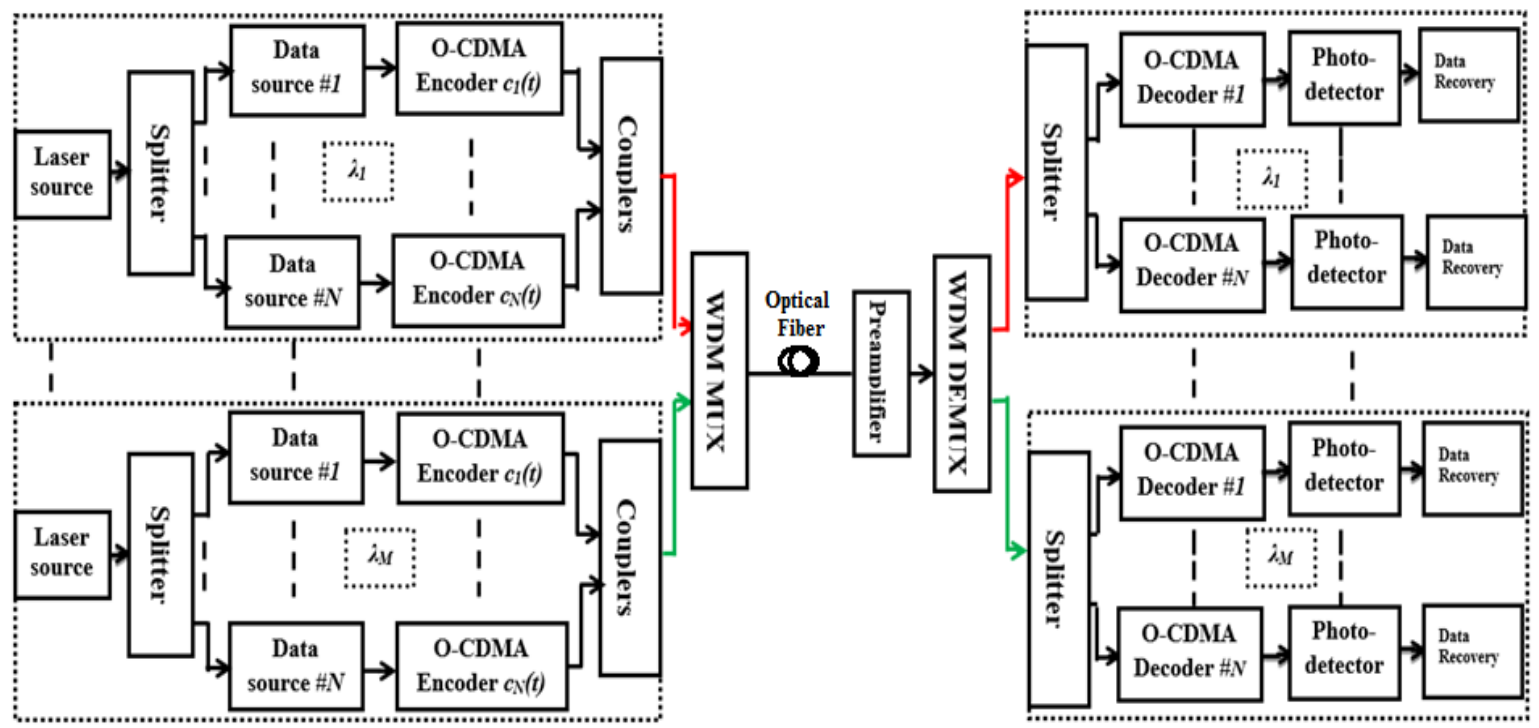

Figure 1. Block diagram of the hybrid system 


\section{FWM IN THE OPTICAL CDMA-DWDM HYBRID SYSTEM}

FWM phenomenon takes place, whenever three different wavelengths propagate inside the fiber. They interact with each other and generate new wavelengths that may coincide with the original wavelengths; the FWM power in the hybrid system due to the interaction of frequencies $f_{p}, f_{q}$ and $f_{r}$ can be expressed as in [23]:

$$
P_{p q r}=\frac{\eta}{9}(d \gamma)^{2} P_{p} P_{q} P_{r}\left[\frac{\left(1-e^{-\alpha L}\right)^{2}}{\alpha^{2}}\right] e^{-\alpha L}
$$

Where, $\eta$ is the FWM efficiency, $d$ is the degeneracy factor, and its value is 1 when $f_{i}=f_{j}$, and 2 when $f_{i} \neq f_{j}, \gamma$ is the nonlinearity coefficient, $L$ is the transmission length, $P_{i}, P_{j}, P_{k}$, are the transmitted power per wavelength/channel, each wavelength is shared with $N$ optical CDMA users, therefore, the transmitted power of each user (e.g., in wavelength $p$ ) is $\frac{P_{p}}{N}$ where the transmitted power per chip is $\frac{P_{p}}{F_{m} N}$; $F_{m}$ is the signature sequence length and $\alpha$ is the attenuation of the fiber. The nonlinearity coefficient is expressed as $\gamma=\frac{2 \pi n_{2}}{\lambda A_{e f f}}, n_{2}$ is the fiber nonlinear refractive index, which is related to the third nonlinear susceptibility $x_{111}$ given by $n_{2}=\frac{48 \pi^{2}}{c n^{2}} x_{111}$, where $c, n, A_{\text {eff }}$ and $\lambda$, are the vacuum speed of light, the refractive index of the fiber core, the effective area of the fiber core, the vacuum wavelength, respectively. The FWM efficiency $\eta$ depends on the phase mismatching and can be expressed as:

$$
\eta=\frac{\alpha^{2}}{\alpha^{2}+(\Delta \beta)^{2}}\left[1+\frac{4 e^{-\alpha L} \sin ^{2}\left(\frac{\Delta \beta . L}{2}\right)}{\left(1-e^{-\alpha L}\right)^{2}}\right]
$$

The FWM efficiency $\eta$ takes maximum value 1 when $\Delta \beta=0$, where; $\Delta \beta$ refers to the phase mismatch and it can be represented by [23].

$$
\Delta \beta=\frac{2 \pi \lambda^{2}}{c}\left(\Delta f_{i j}\right)\left(\Delta f_{j k}\right)\left[D_{C}+\left(\frac{\lambda^{2}}{2 c}\right)(S D)\left(\Delta f_{p q}+\Delta f_{q r}\right)\right]
$$

where; $D_{C}, S D$ are the chromatic and slope dispersion, respectively.

\section{RESULTS AND DISCUSSIONS}

Considering intensity modulation/direct detection (IM/DD) transmission, the proposed hybrid system consists of 5 wavelengths with wavelength spacing of $0.4 \mathrm{~nm}$ operating at $1559.794 \mathrm{~nm}-1561.419 \mathrm{~nm}$, where each carried 12 optical CDMA user's data. Therefore, 60 optical CDMA users can be accommodated by the proposed hybrid system; the implemented parameters are summarized in Table 1. It has been shown in [24] that the ISI can be mitigated as the interval of the signature optical code is squeezed into $25 \%$ of the bit duration; therefore, such interval is considered in our proposed hybrid system.

Figure 2 shows the average BERs versus the transmitted power for the five DWDM channels where each channel carries 12 optical CDMA users at data rate of $5 \mathrm{~Gb} / \mathrm{s} / \mathrm{user}$. The signals are transmitted over $90 \mathrm{~km}$ of SMF and $15.075 \mathrm{~km}$ of DCF. According to our previous work [24], the best performance of the proposed system when the transmitted power is $18 \mathrm{dBm}$, beyond this value the system performance significantly decreases because of the effect of the intra FWM. In this work, the amplified spontaneous emission (ASE) is neglected in order to have some insight on FWM effects. The spreading of the energy of the bit over the signature sequence code, the FWM is reduced as the energy is distributed over the set of optical sequence code. Hence, good performance of 60 users is achieved. It can be extracted from the results that the position of weights "ones" in the signature sequence code plays crucial role in increasing the nonlinear tolerance in the hybrid system as the longer the distance between the ones of the code the better the performance. The results also revealed that the hybrid system has its best performance when the transmitted 
power is $18 \mathrm{dBm}$. Figure 3 shows the variation of the BERs of random 5 optical CDMA users out of 12 in channel 1 versus the transmitted power. As noted that the BER of user 8 is slightly bigger than those of other users because of the weights' positions in the optical sequence code are close to each other.

In reference [25], the authors proposed hybrid WDM-OCDMA network employing $60 \mathrm{~Gb} / \mathrm{s}$ nonreturn to zero/differential quadrature phase shift keying (NRZ/DQPSK) orthogonally modulated data signal operating over $100 \mathrm{~km}$ (SMF+DCF), which was capable of supporting 32 users carried by 4 wavelengths. In comparison with reference [25], we managed to implement a hybrid system over $105.075 \mathrm{~km}$ (SMF+DCF), which is capable of accommodating 60 optical CDMA users carried by only 5 wavelengths with channel spacing of $0.4 \mathrm{~nm}$.

Table 1. The Performance of the proposed hybrid system

\begin{tabular}{cccc}
\hline Parameter & Value & Parameter & Value \\
\hline Number of channels & $\mathrm{M}=5$ & Attenuation for SMF & $0.2 \mathrm{~dB} / \mathrm{km}$ \\
Number of users in each channel & $\mathrm{N}=12$ & Dispersion for SMF & $16.75 \mathrm{ps} / \mathrm{nm} . \mathrm{km}$ \\
Length and weight of the sequence code & $\mathrm{F}=24, \quad \mathrm{w}=2$ & Dispersion slope for SMF & $0.075 \mathrm{ps} / \mathrm{nm}^{2} . \mathrm{km}$ \\
Input transmitted power to the fiber & $18 \mathrm{dBm}$ & Cross effective area for DCF & $22 \mathrm{um}{ }^{2}$ \\
Total channel length & $105.075 \mathrm{~km}(90 \mathrm{~km} \mathrm{SMF}$ & Dispersion for DSF & $-100 \mathrm{ps} / \mathrm{nm} . \mathrm{km}$ \\
& $+15.075 \mathrm{~km} \mathrm{DCF})$ & & \\
Preamplifier gain & $5 \mathrm{~dB}$ & Dispersion slope for DSF & $-0.45 \mathrm{ps} / \mathrm{nm}^{2} . \mathrm{km}$ \\
Channel spacing & $0.4 \mathrm{~nm}^{2}$ & Attenuation for DCF & $0.5 \mathrm{~dB} / \mathrm{km}$ \\
Cross effective area for SMF & $80 \mathrm{um}^{2}$ & Data rate per channel & $60 \mathrm{~Gb} / \mathrm{s}$ \\
\hline
\end{tabular}

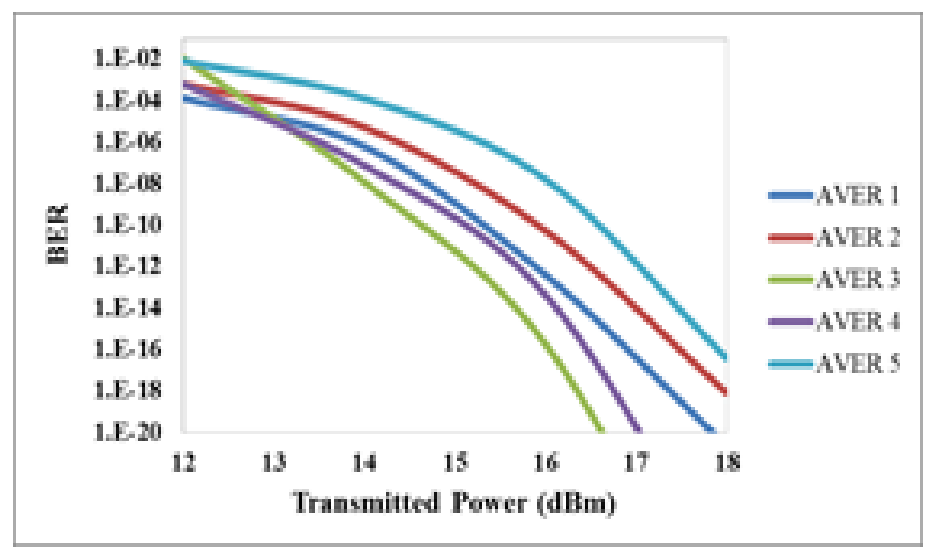

Figure 2. The average BERs versus transmitted power for the five DWDM channels with 0.4nm spacing

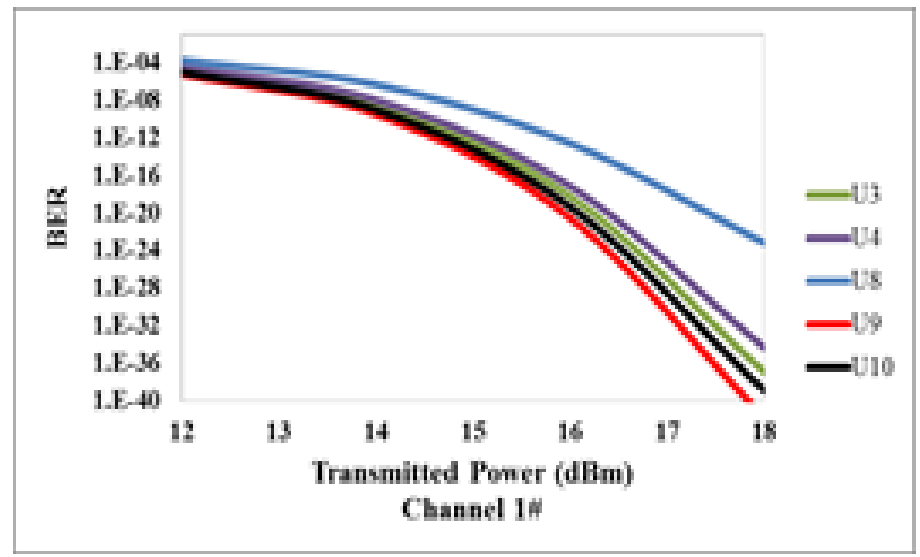

Figure 3. BERs versus transmitted power for 5 random optical CDMA users out of 12 carried in channel 1 


\section{CONCLUSION}

This paper reported a hybrid system of 12 optical CDMA users x 5 DWDM with channel spacing of $0.4 \mathrm{~nm}$ at $60 \mathrm{~Gb} / \mathrm{s} /$ wavelength. In the proposed system the inter-symbol interference (ISI) is mitigated by squeezing the interval of optical sequence code into $25 \%$ of the bit duration, while the multiple access interference (MAI) is minimized due to the use of MD sequence code. The results revealed that the CDMA technology could be used to reduce the effect of FWM in the proposed hybrid system. It is found that the optimum values of transmitted power and transmission distance are $18 \mathrm{dBm}$ and $105.075 \mathrm{~km}$ respectively. In future work, an electro optic phase modulator (EOPM) will be implemented to enhance the nonlinear tolerance of the proposed hybrid system.

\section{ACKNOWLEDGEMENTS}

This work was supported by Multimedia University (Malaysia), project SAP ID: MMUI/160092.

\section{REFERENCES}

[1] K. Kitayama, Xu Wang and Naoya Wada, "OCDMA over WDM PON-solution path to gigabit-symmetric FTTH", J. Lightwave Tech, vol. 24, no. 4, pp. 1654-1662, 2006.

[2] Wang X, Wada N, Miyazaki T, Cincotti G, Kitayama K. Field Trial of 3-WDM $\times 10-O C D M A \times 10.71-G b / s$ Asynchronous WDM/DPSK-OCDMA Using Hybrid E/D without FEC and Optical Thresholding. J. Lightwave Tech., vol. 25, no. 1, pp. 207-215., 2007.

[3] He Chen, Shilin Xiao, Min Zhu, Jie Shi, Meihua Bi"Hybrid WDMA/OCDM system with the capability of encoding multiple wavelength channels by employing one encoder and one corresponding optical code", Chinese Optics Letters, vol. 8, no. 8, pp. 745-748, 2010.

[4] Y. Choi, M. Hanawa, X. Wang and C. Park, "Upstream Transmission of WDM/OCDM-PON in a Loop-Back Configuration With Remotely Supplied Short Optical Pulses", Journal of Optical Communications and Networking, vol. 5, no. 3, p. 183, 2013.

[5] J. Huang, T. Nieh and K. Chen, "Structuring waveguide-grating-based wavelength-division multiplexing/optical code division multiple access network codecs over topology of concentric circles", Optical Engineering, vol. 52, no. 1, p. 015006, 2013

[6] F. Hou and M. Yang, "The analysis of system performance of WDM/OCDMA-PON based on DQPSK", Optik, vol. 125, no. 15, pp. 4150-4153, 2014.

[7] H. Mrabet, I. Dayoub and R. Attia, "Comparative study of 2D-OCDMA-WDM system performance in 40-Gb/s PON context", IET Optoelectronics, vol. 11, no. 4, pp. 141-147, 2017.

[8] M. Eghbal and M. Shadaram, "Expandable high-capacity wavelength division multiplex-over-optical code division multiple access millimeter wave radio-over-fiber system", Optical Engineering, vol. 58, no. 01, p. 1, 2019.

[9] M. Rana, "Dense Wavelength Division Multiplexing Optical Network System", International Journal of Electrical and Computer Engineering (IJECE), vol. 2, no. 2, pp. 203-206, 2011.

[10] V. Pechenkin and I. Fair, "On Four-Wave Mixing Suppression in Dispersion-Managed Fiber-Optic OFDM Systems With an Optical Phase Conjugation Module", J. Lightwave Tech., vol. 29, no. 11, pp. 1678-1691, 2011.

[11] L. Li, W. Jian-yi, L. Hong-an and Z. Xiu-tai, "Analysis Modulation Formats in DWDM Transmission System", TELKOMNIKA Indonesian Journal of Electrical Engineering, vol. 11, no. 1, pp. 536-543, 2012.

[12] F. Xiancheng, L. Xiaopeng and S. Qinghua, "Research on OSNR and BER of 40G DWDM system on DRZ", TELKOMNIKA Indonesian Journal of Electrical Engineering, vol. 11, no. 2, pp.948-953, 2012.

[13] L. Li, W. Jian-yi, Z. Xiu-tai and L. Hong-an, "Research on Mixed Data Rate and Format Transmission in WDM Networks", TELKOMNIKA (Telecommunication, Computing, Electronics and Control), vol. 11, no. 1, pp. 127-136, 2013.

[14] L. Li, C. Yan-Tao, Z. Ji-Jun, L. Zhi-Rui and C. Wan-Li, "Research on the Modulation Performance in GPON System", TELKOMNIKA Indonesian Journal of Electrical Engineering, vol. 12, no. 10, pp. 7304-7310, 2014.

[15] C. Mounia, A. Otman, A. Badiaa, C. Fahd, A. Ana Vazquez and E. Mounir, "Gain Flatness and Noise Figure Optimization of C-Band EDFA in 16-channels WDM System using FBG and GFF", International Journal of Electrical and Computer Engineering (IJECE), vol. 7, no. 1, pp. 289-298, 2017.

[16] J. Du, Y. Xu, F. Han and W. Yang, "Impact of quasi-linear pulse evolution on FWM noise in dispersion-managed DPSK DWDM systems", Optik, vol. 140, pp. 381-391, 2017.

[17] B. Das, M. Abdullah and N. Mohd Shah, "A New All-Optical Signal Regeneration Technique for 10 GB/S DPSK Transmission System", International Journal of Electrical and Computer Engineering (IJECE), vol. 6, no. 2, pp. 859-869, 2016.

[18] L. Jiang, X. Yuan, Y. Cui, G. Chen, F. Zuo and C. Jiang, "Optical bistability and four-wave mixing in a hybrid optomechanical system", Physics Letters A, vol. 381, no. 38, pp. 3289-3294, 2017.

[19] G. Singh and M. Singh, "Spectral bandwidth-efficient four-wave mixing minimization scheme for C-band dense wavelength division multiplexed system", Optical Engineering, vol. 56, no. 7, p. 076115, 2017.

[20] S. Singh and S. Singh, "On compensation of four wave mixing effect in dispersion managed hybrid WDM-OTDM multicast overlay system with optical phase conjugation modules", Optical Fiber Technology, vol. 38, pp. 160-166, 2017. 
[21] N. Alsowaidi, T. Eltaif, M. Mokhtar and B. Hamida, "Reduction of Four-Wave Mixing in DWDM System using Electro-Optic Phase Modulator", International Journal of Electrical and Computer Engineering (IJECE), vol. 8, no. 4, pp. 2384-2389, 2018.

[22] Safar A, Aljunid S, Arief A, Nordin J, Saad N. Minimizing correlation effect using zero cross correlation code in spectral amplitude coding optical code division multiple access. Optical Review. 2012;19(1):20-24.

[23] Agrawal, G. Nonlinear fiber optics. $5^{\text {th }}$ ed., (Amsterdam: Academic Press, 2013, pp. 397-457.

[24] Alsowaidi N, Eltaif T, Mokhtar M. Suppression of Inter and Intra Channel Four Wave Mixing effect in Optical CDMA over DWDM Hybrid System. Chinese optics. 2019; 9 (1), in press.

[25] Singh S, Singh S. Performance analysis of spectrally encoded hybrid WDM-OCDMA network employing optical orthogonal modulation format against eavesdropper. AEU - International Journal of Electronics and Communications. 2017;82:492-501.

\section{BIOGRAPHIES OF AUTHORS}

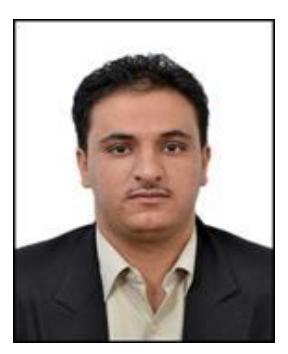

Naif Alsowaidi received the B.Eng. degree (with honors) in communication engineering from International Islamic University Malaysia (IIUM), in 2012 and the M.Sc. degree. in engineering science from Multimedia University (MMU), Malaysia, in 2015, currently he is a Ph.D. candidate at Multimedia University. His current research interests include optical communication, Optical CDMA systems, and nonlinear optics.

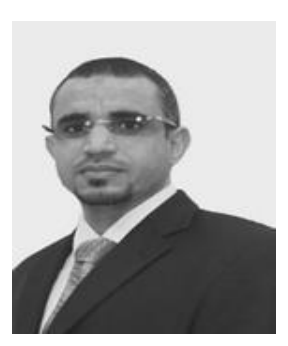

Tawfig Eltaif received his BSc degree in communication engineering from University of Tripoli, Libya, 2003, and MSc degree in microelectronics and Ph.D degree in micro-engineering \& nanoelectronics from National University of Malaysia, Malaysia, 2005, 2009, respectively.

He joined Photronix Technologies Company, 2008-2011, as a product and research engineer. Since 2012 he has been a senior lecturer at Multimedia University. His current research is focused on optical communication, optical frequency comb, FBG, and optical amplifiers.

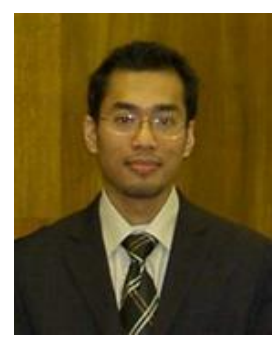

Mohd Ridzuan Mokhtar received his Ph.D degree in optoelectronics from the University of Southampton, UK, in 2005. He is professor at the Faculty of Engineering, Multimedia University, Malaysia. He became a research fellow with City University London, UK, for a year in 2009, sponsored by the Technology Strategy Board in the UK. He has been the director of research and collaboration at Multimedia University since 2011. His main research interests include fiber optic sensors and optical communications. 